\title{
Conformational Energy and Unperturbed Chain Dimension of Polypeptide Copolymers
}

\author{
Seiji TANaka and Akio Nakajima* \\ Department of Polymer Chemistry, Kyoto University, Sakyo-ku, Kyoto Japan.
}

(Recived March 20, 1971)

\begin{abstract}
Copolypeptides composed of two components among L-alanine, glycine, $N$-methylglycine, and $N$-methyl-L-alanine, and $\mathrm{D}, \mathrm{L}$-copolypeptides composed either of D-alanine and L-alanine or of $N$-methyl-D-alanine, $N$-methyl-L-alanine were theoretically investigated for conformational energies and unperturbed average chain dimensions, assuming trans conformation both for amide and imide bonds.

The characteristic ratios of copolymers were calculated as a function of sequence probability that a residue of the minor component is followed by a residue of the same kind at varied copolymer compositions. For equimolar copolymers, the effect of the degrees of polymerization was also investigated. In racemic D,L-copolymers, the chain dimensions are predicted as markedly dependent on the isotacticity of the polymer. The remarkable effect of $N$-methylalanine residue on the chain dimension was also discussed.
\end{abstract}

KEY WORDS Copolymer / Polypeptide / D,L-Copolypeptide / Racemic Copolymer / Glycine / Alanine / $N$-methylglycine / $N$-methylalanine/ Characteristic Ratio / Chain Conformation /

In our preceding paper, ${ }^{1,14}$ the characteristic ratios of polypeptide homopolymers such as poly(L-alanine), polyglycine, poly $(N$-methyl-Lalanine) and poly $(N$-methylglycine), were theoretically investigated.

With regard to polypeptipe copolymers, so far, theoretical calculations on the chain dimension have been performed on random glycinealanine-type copolymers, ${ }^{2,3}$ D,L-copolymers ${ }^{2,3}$ having alanine-type side chains, and copolymers $^{2,3}$ containing proline residues.

The advantage in conformation calculations of polypeptides is that the chain can be treated as a semiindependent rotation model chain, ${ }^{3-5}$ whose conformation is described by a function only of $\phi$ and $\phi$, the rotational angles around $\mathrm{N}_{i}-\mathrm{C}_{i}{ }^{\alpha}$ and $\mathrm{C}_{i}{ }^{\alpha}-\mathrm{C}_{i}{ }^{\alpha}$ bonds adjoining $\mathrm{C}_{i}{ }^{\alpha}$ (see Figure 1). The semiindependent rotation model is also valid for copolypeptides, and enables us to calculate explicitly the characteristic ratio based on configurational and conformational considerations on the copolypeptide chains, without using the Monte Carlo calculation.

In this paper, we shall deal with the characteristic ratios for copolypeptides composed of

\footnotetext{
* To whom correspondence should be addressed.
}

two components out of L-alanine'abbreviated as Ala), glycine(Gly), $N$-methylglycine(NMG), and $N$-methyl-L-alanine(NMA), and for D,L-copolypeptides composed either of D-alanine(D-Ala) and L-alanine(L-Ala) or of $N$-methyl-D-alanine(DNMA) and $N$-methyl-L-alanine(L-NMA), by assuming trans conformation for all the amide and imide bonds and employing the same parameters and potential functions as used in a preceding paper. ${ }^{1}$

With regard to $N$-methyl-L-alanine, the characteristic ratio of the homopolymer obtained in a preceding paper $^{1}$ was as small as ca. 0.6. Moreover, the conformational energy contour diagram obtained there also suggests that the NMA sequence in homopolymer and copolymer chains is treated as a helix rather than a random coil, though the experimental result is not known. For these reasons, in this paper, the NMA sequence is treated not only as a random coil but also as a helix.

\section{CONFORMATIONAL ENERGY}

Molecular Structure and Configuration of Copolypeptides 


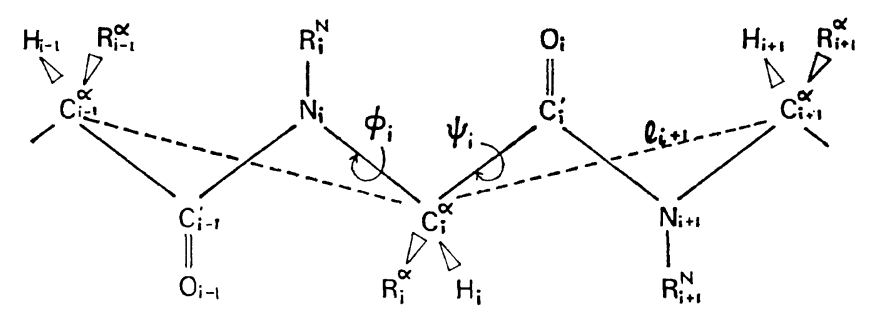

Figure 1. Fully extended copolypeptide chain. Either $\mathbf{R}^{N}$ or $\mathbf{R}^{\alpha}$ represents a hydrogen atom or a methyl group. The broken lines denote virtual bonds.

In Figure 1, a portion of a polypeptide chain, including the $i$-th amino acid residue and parts of both the $(i-1)$-th and the $(i+1)$-th amino acid residues, is represented in its fully extended trans conformation, where $\mathrm{R}_{i}{ }^{\mathrm{N}}$ and $\mathrm{R}_{i}{ }^{\alpha}$ denote, respectively, the substituents attached to the nitrogen atom $\mathrm{N}_{i}$ and to the $\alpha$-carbon atom $\mathrm{C}_{i}{ }^{\alpha}$ of the $i$-th residue. This figure, which is the same as in a preceding paper, ${ }^{1}$ is also adopted for copolypeptides by regulating the kind of the substituents assigned for $\mathbf{R}_{i}{ }^{\mathrm{N}}, \mathbf{R}_{i}{ }^{\alpha}, \mathbf{R}_{i+1}^{\mathrm{N}}$, etc. As is obvious from Figure 1, the conformational energy $V\left(\phi_{i}, \phi_{i}\right)$ of the $i$-th residue in a copolymer chain depends on the kind of the $(i+1)$-th residue but not on that the $(i-1)$-th residue. Concretely, in the $(i+1)$-th residue the substituent $\mathbf{R}_{i+1}^{\mathrm{N}}$ participates to determine $V\left(\dot{\phi}_{i}\right.$, $\left.\phi_{i}\right)$, but in the $(i-1)$-th residue only the atom $\mathbf{O}_{i-1}$ plays the role. Thus, the kind of the $(i-$ $1)$-th residue does not affect $V\left(\phi_{i}, \phi_{i}\right)$. In conclusion, the conformational energy $V\left(\phi_{i}, \phi_{i}\right)$ of copolypeptides is explicity defined by $\mathbf{R}_{i}{ }^{\mathrm{N}}, \mathbf{R}_{i}{ }^{\alpha}$, and $\mathbf{R}_{i+1}^{\mathrm{N}}$. If we represent $-\mathrm{CH}_{3}$ group and the hydrogen atom attached either to the nitrogen or to the $\alpha$-carbon atom by $\mathrm{R}$ and $\mathrm{H}$, respectively, then all the copolypeptide chains composed of two components out of Ala, Gly, NMG, and NMA are constructed by taking into account the eight kinds of combination of $\left(\mathbf{R}_{i}{ }^{\mathrm{N}} \cdot \mathbf{R}_{i}{ }^{\alpha} \cdot \mathbf{R}_{i+1}^{\mathrm{N}}\right)$ shown in Table I. For example, the configurations given either by Ala-NMG or by Ala-NMA are equally represented by $(\mathbf{H} \cdot \mathbf{R} \cdot \mathbf{R})$. As mentioned in a previous paper, ${ }^{13}$ $\mathrm{R}$ may be regarded as not only the $-\mathrm{CH}_{3}$ group but also the $-\mathrm{CH}_{2}-\mathrm{R}^{\prime}$ group.

Hereafter, the conformational energy $V\left(\phi_{i}, \phi_{i}\right)$ defined as a function of $\phi_{i}$ and $\phi_{i}$ is designated for copolypeptides as $V_{\left(\mathrm{R}_{i}^{\mathrm{N}}, \mathrm{R}_{i}{ }^{\alpha}, \mathrm{R}_{i+1}^{\mathrm{N}}\right)}\left(\phi_{i}, \phi_{i}\right)$,
Table I. Configurations in copolypeptide chains<smiles>[R12][Y10]([H])=O</smiles> 
taking into account the combinations shown in Table I; e.g., $V_{(\mathrm{RRR})}\left(\phi_{i}, \phi_{i}\right)$ for either the NMA -NMA or the NMA-NMG configuration.

As mentioned in a preceding paper, ${ }^{1}$ the conformational energy for each combination is given by eq 1 , where $V_{(\mathrm{T})}\left(\phi_{i}\right)$ and $V_{(\mathrm{T})}\left(\phi_{i}\right)$ are the intrinsic torsional potentials energy $V_{(\mathrm{W}) j k}\left(\phi_{i}\right.$, $\phi_{i}$ ) is the van der Waals interaction energy between nonbonded atoms $j$ and $k$, and the last term concerns the dipole-dipole interaction energy between amide and amide, amide and imide, imide and amide, or imide and imide dipoles.

$$
\begin{aligned}
& V_{\left(\mathrm{R}_{i}^{\mathrm{N}}, \mathrm{R}_{i}{ }^{\alpha}, \mathrm{R}_{i+1}^{\mathrm{N}}\right)}\left(\phi_{i}, \phi_{i}\right) \\
& =V_{(\mathrm{T})}\left(\phi_{i}\right) \\
& +V_{(\mathrm{T})}\left(\phi_{i}\right)+\sum_{j k} V_{(\mathrm{W}) j k}\left(\phi_{i}, \phi_{i}\right) \\
& \quad+\sum_{j k} V_{(\mathrm{D}) j k}\left(\phi_{i}, \phi_{i}\right)
\end{aligned}
$$

Numerical values of molecular parameters, appear in the Lennard-Jones function, and the partial charges on amide and imide groups, and the bond coordinate systems, used in this calculation, are the same as those used in a preceding paper. ${ }^{1}$

The contribution from the term $\sum V_{(\mathrm{D}) j_{l}}\left(\phi_{i}, \phi_{i}\right)$ to the total conformational energy $V\left(\phi_{i}, \psi_{i}\right)$ is shown in Figures 2 and 3, respectively, for the amide-imide and imide-amide dipole interaction. Those for the imide-imide ${ }^{1}$ and amide - amide $^{6}$ dipole interactions are not reproduced here, because they have already been mentioned

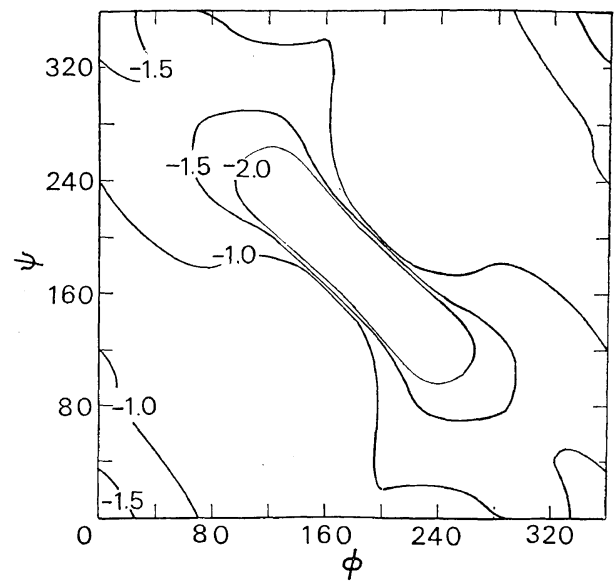

Figure 2. Contour diagram of nearest neighbor imide-amide dipole interaction energy, $\sum V_{(\mathrm{D}) j k}(\phi$, $\phi)$, for configuration (RRH) or (RHH). Numerals on the contours are the absolute energy values.

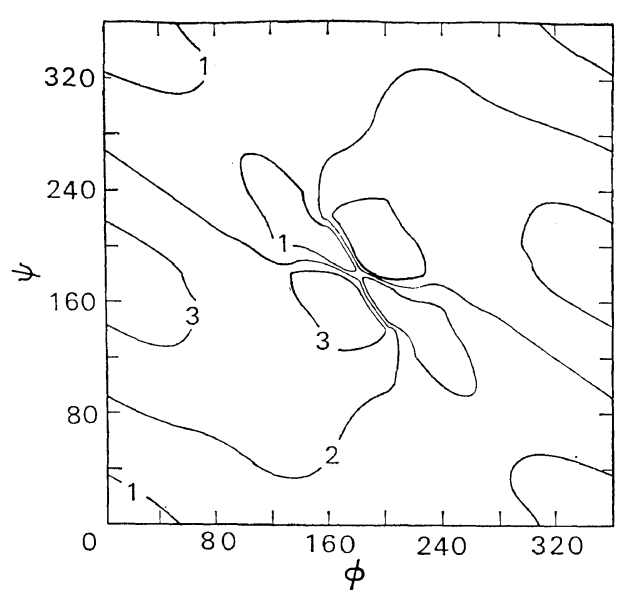

Figure 3. Contour diagram of nearest neighbor amide-imide dipole interaction energy, $\sum V_{(D) j k}(\phi$, $\phi)$, for configuration (HRR) or (HHR). Numerals on the contours are the absolute energy values.

elsewhere. The numerals on the contours in Figures 2 and 3 denote the absolute energy values. To calculate $V_{(\mathrm{HRR})}\left(\phi_{i}, \phi_{i}\right)$ or $V_{(\mathrm{HHR})}\left(\phi_{i}\right.$, $\left.\phi_{i}\right)$ Figure 2 should be used, while Figure 3 is to be used for calculation of $V_{(\mathrm{RRH})}\left(\phi_{i}, \phi_{i}\right)$ or $V_{(\mathrm{RHH})}\left(\phi_{i}, \phi_{i}\right)$.

\section{Conformational Energy Contour Diagrams}

From eq 1, the conformational energy $V_{\left(\mathrm{R}_{i}^{\mathrm{N}}, \mathrm{R}_{i}{ }^{\alpha}, \mathrm{R}_{i+1}^{\mathrm{N}}\right)}\left(\phi_{i}, \phi_{i}\right)$ was calculated at $20^{\circ}$ intervals from $0^{\circ}$ to $340^{\circ}$ for $\phi_{i}$ and $\phi_{i}$. The contour diagram of $V_{(\mathrm{RRR})}\left(\phi_{i}, \phi_{i}\right)$ for the combination NMA-NMG (i.e., NMA succeeded by $\mathrm{NMG}$ ) is the same as that for NMA-NMA (Figure 6 in ref 1 ) which has already been obtained $^{1}$ NMA-homopolymer. Analogously, the contour diagrams for Gly-Ala, Ala-Gly, and NMG-NMA are, respectively, the same as those for Gly-Gly, Ala-Ala, and NMG$\mathrm{NMG}$; those for $V_{(\mathrm{HHH})}\left(\phi_{i}, \phi_{i}\right), V_{(\mathrm{HRH})}\left(\phi_{i}, \phi_{i}\right)$ and $V_{(\mathrm{RHR})}\left(\phi_{i}, \phi_{i}\right)$ are, respectively, represented in Figures 5, 4, and 7 in ref 1.

The conformational energies for NMA succeeded by either Ala or Gly, for Ala succeeded by either NMG or NMA, for NMG succeeded by either Ala or Gly, and for Gly succeeded by either NMG or NMA are, respectively, represented by $V_{(\mathrm{RRH})}\left(\phi_{i}, \phi_{i}\right), V_{(\mathrm{HRR})}\left(\phi_{i}, \phi_{i}\right)$, $V_{(\mathrm{RHH})}\left(\phi_{i}, \phi_{i}\right)$, and $V_{(\mathrm{HHR})}\left(\phi_{i}, \phi_{i}\right)$, as is obvious from Table I. 


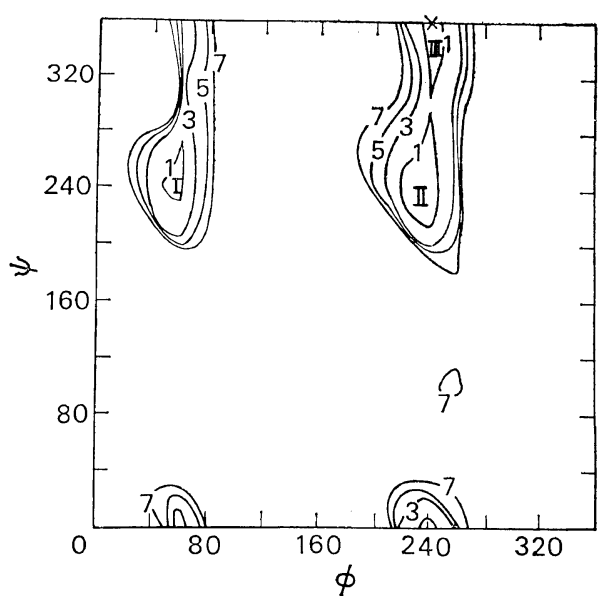

Figure 4. Contour diagram of total conformational energy $V_{(\mathrm{RRH})}(\phi, \phi)$ for NMA-Ala, or NMAGly. The absolute minimum at $\times$ corresponds to $-0.39 \mathrm{kcal} / \mathrm{mol}$; the contour interval is $1 \mathrm{kcal} /$ mol of peptide units relative to the minimum.

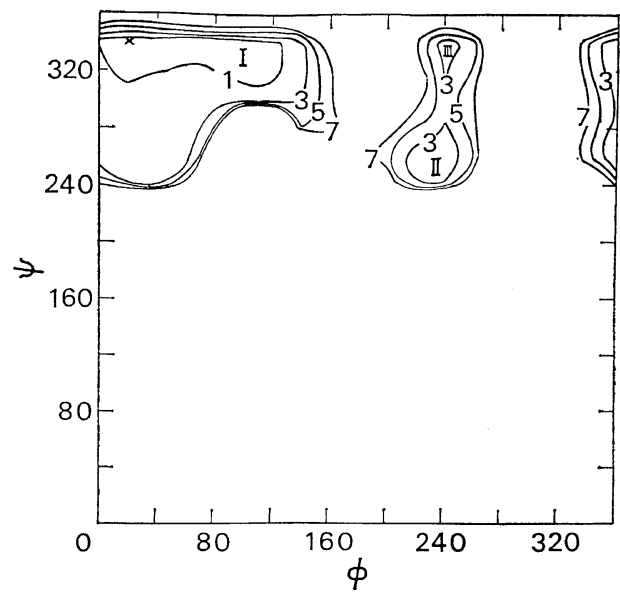

Figure 5. Contour diagram of total conformational energy $V_{(\mathrm{HRR})}(\phi, \phi)$ for Ala-NMG, or AlaNMA. The absolute minimum at $\times$ corresponds to $1.25 \mathrm{kcal} / \mathrm{mol}$; contours are drawn as in Figure 4.

The conformational energy contour diagrams for $V_{(\mathrm{RRH})}\left(\phi_{i}, \phi_{i}\right), V_{(\mathrm{HRR})}\left(\phi_{i}, \phi_{i}\right), V_{(\mathrm{RHH})}\left(\phi_{i}, \phi_{i}\right)$, and $V_{\left(\mathrm{HRR}_{1}\right)}\left(\phi_{i}, \phi_{i}\right)$ are, respectively, indicated in Figures 4-7. The lowest energy positions are denoted by the symbol $x$, and the contours are drawn by the energy levels relative to the lowest energy value. Relatively stable regions

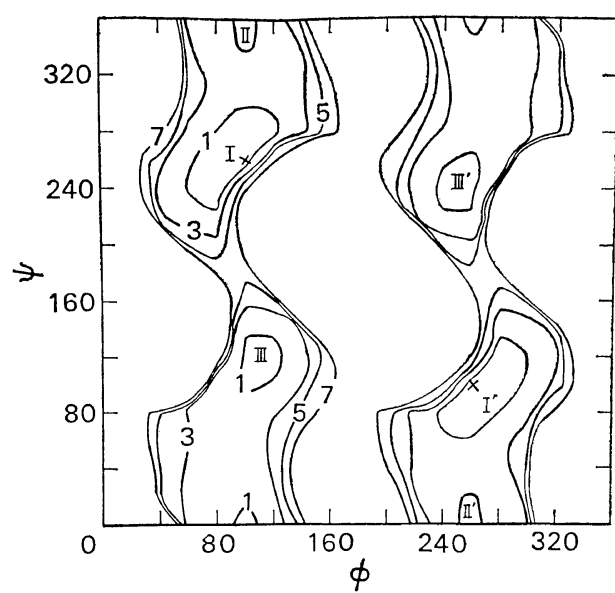

Figure 6. Contour diagram of total conformational energy $V_{(\mathrm{RHH})}(\phi, \phi)$ for $\mathrm{NMG}-\mathrm{Ala}$, or $\mathrm{NMG}$ -Gly. The absolute minima at $\times$ correspond to $-4.14 \mathrm{kcal} / \mathrm{mol}$; contours are drawn as in Figure 4.

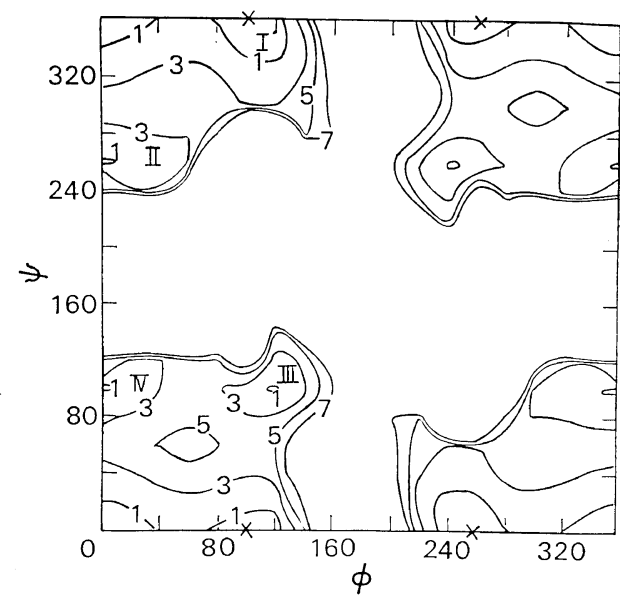

Figure 7. Contour diagram of total conformational energy $V_{(\mathrm{HHR})}(\phi, \phi)$ for Gly-NMG, or GlyNMA. The absolute minima at $\times$ correspond to $-1.34 \mathrm{kcal} / \mathrm{mol}$; contours are drawn as in Figure 4.

in each diagram are designated as regions I, II, III, etc. In Table II are shown the most stable conformations as given by $(\phi, \phi)$ in each region and their energy absolute value. The conformational energies, without dipole interaction terms [i.e., $\left.\Sigma V_{(\mathrm{D}) j k}\left(\phi_{i}, \phi_{i}\right)=0, \varepsilon=\infty\right]$, are shown in column 6 . Comparison of column 6 with column 5 may afford data on the dipolar interaction. 
Table II. Low energy conformations on the energy contour diagrams

\begin{tabular}{|c|c|c|c|c|c|}
\hline \multirow{2}{*}{$\begin{array}{c}\text { Configuration } \\
\left(\mathbf{R}_{i}{ }^{\mathrm{N}}, \mathbf{R}_{i}^{\alpha}, \mathbf{R}_{i+1}^{\mathrm{N}}\right)\end{array}$} & \multirow{2}{*}{$\begin{array}{l}\text { Regions designated } \\
\text { on the contour } \\
\text { diagrams, Figures } 4-7\end{array}$} & \multicolumn{2}{|c|}{$\begin{array}{l}\text { Rotational angles } \\
\text { (in degree) }\end{array}$} & \multicolumn{2}{|c|}{$\begin{array}{l}\text { Absolute conformational energy, } \\
V_{\left(\mathrm{R}_{i} \mathrm{i}^{\mathrm{N}}, \mathrm{R}_{i}^{\alpha}, \mathrm{R}_{i+1}^{\mathrm{N}}\right)}(\phi, \phi) \\
\text { in } \mathrm{kcal} / \mathrm{mol}\end{array}$} \\
\hline & & $\phi$ & $\phi$ & $\begin{array}{l}\text { including } \\
V_{(\text {D })}(\phi, \phi) \\
\text { in eq } 1\end{array}$ & $\begin{array}{l}\text { excluding } \\
V_{(\text {D })}(\phi, \phi) \\
\text { in eq } 1\end{array}$ \\
\hline$(\mathrm{RRH})$ & $\left\{\begin{array}{l}\text { I } \\
\text { II } \\
\text { III }\end{array}\right.$ & $\begin{array}{r}60 \\
240 \\
240\end{array}$ & $\begin{array}{r}260 \\
240 \\
0\end{array}$ & $\begin{array}{r}0.3 \\
-0.3 \\
-0.4\end{array}$ & $\begin{array}{l}0.0 \\
0.1 \\
0.4\end{array}$ \\
\hline (HRR) & $\left\{\begin{array}{l}\mathrm{I} \\
\text { III }\end{array}\right.$ & $\begin{array}{r}20 \\
240 \\
240\end{array}$ & $\begin{array}{l}340 \\
260 \\
340\end{array}$ & $\begin{array}{l}1.3 \\
2.4 \\
2.8\end{array}$ & $\begin{array}{r}-0.1 \\
-0.4 \\
0.8\end{array}$ \\
\hline$(\mathrm{RHH})$ & $\left\{\begin{array}{l}\mathbf{I}\left(\mathbf{I}^{\prime}\right) \\
\mathbf{I I}\left(\mathbf{I I}^{\prime}\right) \\
\mathbf{I I I}\left(\mathbf{I I I}^{\prime}\right)\end{array}\right.$ & $\begin{array}{l}100(260) \\
100(0) \\
100(260)\end{array}$ & $\begin{array}{r}260(100) \\
0(260) \\
120(240)\end{array}$ & $\begin{array}{l}-4.1 \\
-3.3 \\
-3.8\end{array}$ & $\begin{array}{l}-3.0 \\
-2.8 \\
-3.6\end{array}$ \\
\hline (HHR) & $\left\{\begin{array}{l}\mathbf{I}\left(\mathbf{I}^{\prime}\right) \\
\text { II }\left(\mathbf{I I}^{\prime}\right) \\
\mathbf{I I I}\left(\mathbf{I I I}^{\prime}\right) \\
\mathbf{I V}\left(\mathbf{I V}^{\prime}\right)\end{array}\right.$ & $\begin{array}{r}100(260) \\
0(360) \\
120(240) \\
0(360)\end{array}$ & $\begin{array}{r}0(360) \\
260(100) \\
100(260) \\
100(260)\end{array}$ & $\begin{array}{l}-1.3 \\
-0.5 \\
-0.5 \\
-0.5\end{array}$ & $\begin{array}{l}-3.1 \\
-2.9 \\
-3.3 \\
-2.9\end{array}$ \\
\hline
\end{tabular}

\section{UNPERTURBED CHAIN DIMENSION}

Arrangement of monomer units in a copolypeptide chain is characterized by the polymerization mechanism. Here we are concerned with A,B-copolypeptides constructed according to the Markoffian process. With a Markoffian chain, the reactivity of the $i$-th monomer unit depends on the kind of the $(i-1)$-th unit, i.e., the relative reactivities of $A$ and $B$ monomer units depend only on the kind of terminal unit of the growing chain. ${ }^{7}$ Let $p_{\mathrm{AB}}$ be the conditional probability that an A unit is succeeded by a B unit, and let us assume that $p_{\mathrm{AB}}$, together with $p_{\mathrm{AA}}, p_{\mathrm{BA}}$ and $p_{\mathrm{BB}}$ similarly defined, are constant in the course of the polymerization process. Then the matrix $\boldsymbol{P}$ of sequence probability is given by eq 2 .

$$
\boldsymbol{P}=\left[\begin{array}{ll}
p_{\mathrm{AA}} & p_{\mathrm{AB}} \\
p_{\mathrm{BA}} & p_{\mathrm{BB}}
\end{array}\right]
$$

For the 1st amino acid residue $(i=1)$, the matrix $\boldsymbol{P}_{1}$ written as

$$
\boldsymbol{P}_{1}=\left[\begin{array}{cc}
p_{\mathrm{A} ; 1} & 0 \\
0 & p_{\mathrm{B} ; 1}
\end{array}\right]
$$

where $p_{\mathrm{A} ; 1}$ and $p_{\mathrm{B} ; 1}$ denote, respectively, the $a$ priori probabilities that the 1st residue is $\mathrm{A}$ and B.

The matrices $\boldsymbol{P}$ and $\boldsymbol{P}_{1}$ are stochastic, hence

$$
\left.\begin{array}{l}
p_{\mathrm{AA}}+p_{\mathrm{AB}}=1 \\
p_{\mathrm{BA}}+p_{\mathrm{BB}}=1 \\
p_{\mathrm{A} ; 1}+p_{\mathrm{B} ; 1}=1
\end{array}\right\}
$$

Further, denoting the $a$ priori probability that the $i$-th residue is an $\mathrm{A}$ or $\mathrm{B}$ unit by $p_{\mathrm{A} ; i}$ or $p_{B ; i}$, we obtain the following relation after the Markoffian process.

$$
\left[\begin{array}{ll}
p_{\mathrm{A} ; i} & p_{\mathrm{B} ; i}
\end{array}\right]\left[\begin{array}{ll}
p_{\mathrm{AA}} & p_{\mathrm{AB}} \\
p_{\mathrm{BA}} & p_{\mathrm{BB}}
\end{array}\right]=\left[\begin{array}{ll}
p_{\mathrm{A} ; i+1} & p_{\mathrm{B} ; i+1}
\end{array}\right]
$$

We now assume that the chain is sufficiently long. Then $p_{\mathrm{A} ; i}$ and $p_{\mathrm{B} ; i}$ are considered to be independent of $i$. Let $p_{\mathrm{A}}$ and $p_{\mathrm{B}}$ be $a$ priori probabilities for $A$ and $B$, respectively, averaged over all the units. Then

$$
p_{\mathrm{A}} p_{\mathrm{AA}}+p_{\mathrm{B}} p_{\mathrm{BA}}=p_{\mathrm{A}}
$$

These a priori probabilities and conditional probabilities are related to such quantities which are experimentally obtained as the monomer frequencies $F_{A}$ and $F_{B}$ and the dimer frequencies $F_{A A}, F_{A B}$, etc. ${ }^{8}$ In other words, the monomer frequencies are equal to corresponding $a$ priori probabilities, i.e., $\mathrm{F}_{\mathrm{A}}=p_{\mathrm{A}}$ and $\mathrm{F}_{\mathrm{B}}=p_{\mathrm{B}}$. The dimer frequencies are related to the conditional probabilities by the relations $\mathrm{F}_{\mathrm{AA}}=\mathrm{F}_{\mathrm{A}} p_{\mathrm{AA}}, \mathrm{F}_{\mathrm{AB}}=$ $\mathrm{F}_{\mathrm{A}} p_{\mathrm{AB}}, \quad \mathrm{F}_{\mathrm{BA}}=\mathrm{F}_{\mathrm{B}} p_{\mathrm{BA}}$, and $\mathrm{F}_{\mathrm{BB}}=\mathrm{F}_{\mathrm{B}} p_{\mathrm{BB}}$. Thus eq 6 , for example, is written as 


$$
\mathrm{F}_{\mathrm{A}} p_{\mathrm{AA}}+\mathrm{F}_{\mathrm{B}} p_{\mathrm{BA}}=\mathrm{F}_{\mathrm{A}}
$$

Further, among dimer frequencies the relations $F_{A B}=F_{B A}$ and $F_{A A}+F_{A B}+F_{B A}+F_{B B}=1$ are held. Accordingly, Markoffian distribution is described by two independent variables, e.g., if $p_{\mathrm{A}}$ and $p_{\mathrm{AA}}$ are known, then other quantities are given by a function of $p_{\mathrm{A}}$ and $p_{\mathrm{AA}}$ or if $\mathrm{F}_{\mathrm{A}}, \mathrm{F}_{\mathrm{AA}}$, etc., are experimentally known, then conditional probabilities are obtained.

The chain conformations of binary copolypeptides may be classified into two categories, type I and type II; copolymers in which the conformational energy $V\left(\phi_{i}, \phi_{i}\right)$ for the $i$-th unit is not varied by the selection of monomers (A or B) for $(i+1)$-th unit are denoted as type I, while those in which $V\left(\phi_{i}, \phi_{i}\right)$ for the $i$-th unit is varied in accordance with the monomer selection for the $(i+1)$-th unit are denoted as type II. In type I are included the homopolymers as mentioned previously ${ }^{1}$ and copolymers composed of either amide-amide or imideimide sequence. On the other hand, copolymers composed of Ala and NMG, Ala and NMA, Gly and NMG, or Gly and NMA, and copolymers ${ }^{3}$ including prolyl units, are included in type II.

The statistical average $\langle\boldsymbol{T}\rangle$ of the matrix $\boldsymbol{T}$ which transforms virtual bond vector $\boldsymbol{l}_{i+1}$ to $\boldsymbol{l}_{i}$ is given $b^{1}$

$$
\begin{aligned}
& \langle\boldsymbol{T}\rangle \\
& =\frac{\int_{0}^{2 \pi} \int_{0}^{2 \pi} T\left(\phi, \phi, \theta^{\prime \prime \prime}, \xi, \eta\right) \exp [-V(\phi, \phi) / R T] \mathrm{d} \phi \mathrm{d} \psi}{\int_{0}^{2 \pi} \int_{0}^{2 \pi} \exp [-V(\phi, \phi) / R T] \mathrm{d} \phi \mathrm{d} \psi}
\end{aligned}
$$

for the eight kinds of configurations shown in Table I; these are expressed as $\langle\boldsymbol{T}\rangle_{(\mathrm{HHH})}$, $\langle\boldsymbol{T}\rangle_{(\mathrm{HRH})},\langle\boldsymbol{T}\rangle_{(\mathrm{RHR})}$, etc.

According to the theory given by Flory, ${ }^{9,10}$ the characteristic ratio of homopolypeptides with finite chain length is expressed as

$$
\frac{\left\langle R_{0}{ }^{2}\right\rangle}{n l^{2}}=\frac{2}{n l^{2}}\left[\begin{array}{lllll}
1 & 0 & 0 & 0 & 0
\end{array}\right] \prod_{i=1}^{n} G_{i}\left[\begin{array}{l}
0 \\
0 \\
0 \\
0 \\
1
\end{array}\right]
$$

in which

$$
\boldsymbol{G}_{i}=\left[\begin{array}{ccc}
1 & \boldsymbol{l}^{\mathrm{T}}\langle\boldsymbol{T}\rangle & \boldsymbol{l}^{2} / 2 \\
\boldsymbol{0} & \langle\boldsymbol{T}\rangle & \boldsymbol{l} \\
0 & \boldsymbol{0} & 1
\end{array}\right]
$$

When we substitute $\langle\boldsymbol{T}\rangle_{(\mathrm{RHR})}$ in $\langle\boldsymbol{T}\rangle$, we obtain $\left\langle R_{0}{ }^{2}\right\rangle / n l^{2}$ for NMG-homopolymer. ${ }^{1}$

The characteristic ratio for type I copolymers is evaluated by the method reported by Miller, et $a .^{2}{ }^{2}$ For A,B-copolymers, $\langle\boldsymbol{T}\rangle_{\mathrm{A}}$ and $\langle\boldsymbol{T}\rangle_{\mathrm{B}}$ are evaluated from eq 8 and with the matrices $\boldsymbol{G}_{\mathrm{A}}$ and $\boldsymbol{G}_{\mathrm{B}}$ are constructed. Note that for type I copolymers, $\boldsymbol{G}_{\mathrm{A}}=\boldsymbol{G}_{\mathrm{AA}}=\boldsymbol{G}_{\mathrm{AB}}$ and $\boldsymbol{G}_{\mathrm{B}}=\mathrm{G}_{\mathrm{BA}}=$ $\boldsymbol{G}_{\mathrm{BB}}$. Now, introducing the matrix $\boldsymbol{G}$ and matrix $\boldsymbol{P}_{i}$ [which equal to $\boldsymbol{P}$ in eq 2], we construct the matrix $\boldsymbol{K}$ for $i$-th residue,

$$
\boldsymbol{K}=\left[\begin{array}{ll}
p_{\mathrm{AA}} \boldsymbol{G}_{\mathrm{A}} & p_{\mathrm{AB}} \boldsymbol{G}_{\mathrm{B}} \\
p_{\mathrm{BA}} \boldsymbol{G}_{\mathrm{A}} & p_{\mathrm{BB}} \boldsymbol{G}_{\mathrm{B}}
\end{array}\right]
$$

and $K_{1}$ for $i=1$.

$$
\boldsymbol{K}_{1}=\left[\begin{array}{cc}
p_{\mathrm{A} ; 1} \boldsymbol{G}_{\mathrm{A}} & \boldsymbol{0} \\
\boldsymbol{0} & p_{\mathrm{B} ; 1} \boldsymbol{G}_{\mathrm{B}}
\end{array}\right]
$$

Thus the characteristic ratio for this type of A,B-copolypeptide chain is given by

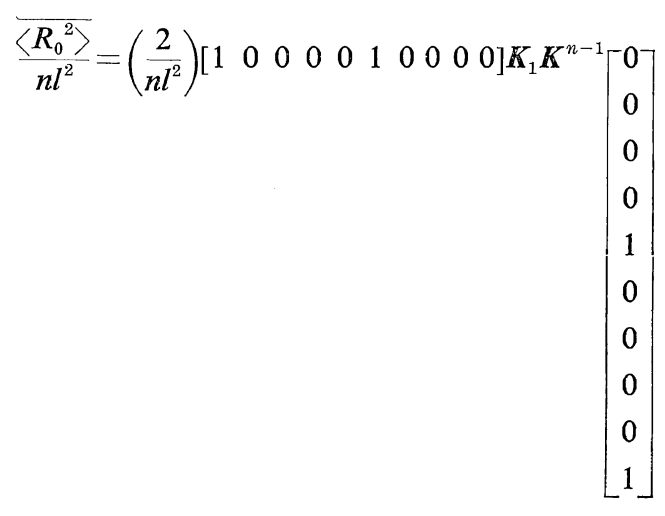

In eq 13 , the bar drawn over $\left\langle R_{0}{ }^{2}\right\rangle$ means the average over all the possible configurations of copolymers obtained by polymerization reaction.

With respect to type II copolymers, since the conformational energy of the $i$-th residue depends on the monomer selection for the $(i+1)$-th residue, $G_{i}$ in eq 10 should be individually constructed for $\mathrm{AA}, \mathrm{AB}, \mathrm{BA}$, and $\mathrm{BB}$, respectively, as $\boldsymbol{G}_{\mathrm{AA}}, \boldsymbol{G}_{\mathrm{AB}}, \boldsymbol{G}_{\mathrm{BA}}$, and $\boldsymbol{G}_{\mathrm{BB}}$. With these matrices $G$ 's, a matrix $K$ analogous to eq 11 should be constructed. However, for type II copolymers, 
the matrices $\boldsymbol{G}$ 's and $\boldsymbol{P}$ introduced should be different from those appearing in eq 11. In this case, the treatment as used by Schimmel, et al. ${ }^{3}$ is necessary. For type II copolymers, the reactivity of the $i$-th residue depends on the kind of the $(i-1)$-th residue, while the conformation of the $i$-th residue depends on the kind of the $(i+1)$-th residue. Accordingly, the matrix $K$ for the $i$-th residue should be constructed after the reaction proceeds to the $(i+$ 1)-th residue. In other words, $\boldsymbol{P}$ introduced in $\boldsymbol{K}$ should be $\boldsymbol{P}_{i+1}$, i.e., $\boldsymbol{P}$ for $(i+1)$-th residue, instead of $\boldsymbol{P}_{i}$, i.e., $\boldsymbol{P}$ for $i$-th residue.

Thus, for the $i$-th residue $(1<i<n)$

$$
\boldsymbol{K}=\left[\begin{array}{ll}
\boldsymbol{G}_{\mathrm{AA}} p_{\mathrm{AA}} & \boldsymbol{G}_{\mathrm{AB}} p_{\mathrm{AB}} \\
\boldsymbol{G}_{\mathrm{BA}} p_{\mathrm{BA}} & \boldsymbol{G}_{\mathrm{BB}} p_{\mathrm{BB}}
\end{array}\right]
$$

and for the 1 st residue $(i=1)$

$$
\boldsymbol{K}_{1}=\left[\begin{array}{cc}
\boldsymbol{E}_{5} p_{\mathrm{A}} & \boldsymbol{0} \\
\boldsymbol{0} & \boldsymbol{E}_{5} p_{\mathrm{B}}
\end{array}\right]
$$

where $\boldsymbol{E}_{5}$ is the unit matrix of the order 5 . For the terminal residue $(i=n)$, the matrix $K_{n}{ }^{*}$ is written as

$$
\begin{aligned}
\boldsymbol{K}_{n}^{*}= & \boldsymbol{K}_{n-1}\left[\begin{array}{ll}
\boldsymbol{G} & \boldsymbol{0} \\
\boldsymbol{O} & \boldsymbol{G}
\end{array}\right] \\
& =\left[\begin{array}{ll}
\boldsymbol{G}_{\mathrm{AA}} p_{\mathrm{AA}} \boldsymbol{G} & \boldsymbol{G}_{\mathrm{AB}} p_{\mathrm{AB}} \boldsymbol{G} \\
\boldsymbol{G}_{\mathrm{BA}} p_{\mathrm{BA}} \boldsymbol{G} & \boldsymbol{G}_{\mathrm{BB}} p_{\mathrm{BB}} \boldsymbol{G}
\end{array}\right]
\end{aligned}
$$

Finally, the characteristic ratio is expressed by

$$
\left.=\left(\frac{2}{n l^{2}}\right)\left[\begin{array}{llllllllll}
1 & 0 & 0 & 0 & 0 & 1 & 0 & 0 & 0 & 0
\end{array}\right] \boldsymbol{K}_{1}{ }^{2}\right\rangle \boldsymbol{K}^{n-2} \boldsymbol{K}_{n}{ }^{*}\left[\begin{array}{l}
0 \\
0 \\
0 \\
0 \\
1 \\
0 \\
0 \\
0 \\
0 \\
1
\end{array}\right]
$$

\section{RESULTS ON NUMERICAL CALCULATION}

Statistical Mechanical Average of Transformation Matrices

The averaged transformation matrices $\langle\boldsymbol{T}\rangle$ 's for eight pairs of configuration were evaluated at an assumed temperature $25^{\circ} \mathrm{C}$ from eq 8 . In practical calculations, the integrals in eq 8 were replaced by summations over $\phi_{i}$ and $\phi_{i}$ from $0^{\circ}$ to $330^{\circ}$ in $30^{\circ}$ intervals. The obtained results are as follows.

$$
\begin{aligned}
& \langle\boldsymbol{T}\rangle_{(\mathrm{RRR})}=\left[\begin{array}{rrr}
0.332 & 0.132 & 0.835 \\
-0.166 & 0.552 & -0.222 \\
-0.557 & -0.027 & 0.223
\end{array}\right] \\
& \langle\boldsymbol{T}\rangle_{(\mathrm{RRH})}=\left[\begin{array}{rrr}
0.340 & 0.156 & 0.492 \\
-0.250 & 0.366 & -0.277 \\
-0.509 & 0.118 & 0.205
\end{array}\right] \\
& \langle\boldsymbol{T}\rangle_{(\mathrm{RRH})}=\left[\begin{array}{ccl}
0.578 & 0.653 & 0 \\
-0.267 & -0.032 & 0 \\
0 & 0 & 0.146
\end{array}\right] \\
& \langle\boldsymbol{T}\rangle_{(\mathrm{HRR})}=\left[\begin{array}{rrr}
0.579 & 0.328 & 0.646 \\
0.043 & -0.658 & 0.232 \\
0.601 & -0.127 & -0.359
\end{array}\right] \\
& \langle\boldsymbol{T}\rangle_{(\mathrm{RHH})}=\left[\begin{array}{ccc}
0.251 & -0.183 & 0 \\
-0.313 & -0.305 & 0 \\
0 & 0 & -0.115
\end{array}\right] \\
& \langle\boldsymbol{T}\rangle_{(\mathrm{HRH})}=\left[\begin{array}{rrr}
0.565 & 0.370 & 0.499 \\
-0.055 & -0.573 & 0.417 \\
0.566 & -0.417 & -0.330
\end{array}\right] \\
& \langle\boldsymbol{T}\rangle_{(\mathrm{HHR})}=\left[\begin{array}{ccc}
0.620 & 0.615 & 0 \\
-0.097 & -0.212 & 0 \\
0 & 0 & -0.047
\end{array}\right] \\
& \langle\boldsymbol{T}\rangle_{(\mathrm{HHH})}=\left[\begin{array}{ccc}
0.368 & -0.004 & 0 \\
-0.163 & -0.392 & 0 \\
0 & 0 & -0.140
\end{array}\right]
\end{aligned}
$$

Eq 18, 20, 23, and 25 are, respectively, the same as eq $11,10,8$, and 9 in ref 1 .

Characteristic Ratios of Copolypeptides

(a) Copolymers of Alanine and $N$-methylglycine. This type of copolymer belongs to type II. The characteristic ratio is obtained from eq 17, in which $K$ is constructed by 
denoting Ala and NMG as A and B, respectively, and writing as

$$
\left[\begin{array}{ll}
\boldsymbol{G}_{\mathrm{AA}} & \boldsymbol{G}_{\mathrm{AB}} \\
\boldsymbol{G}_{\mathrm{BA}} & \boldsymbol{G}_{\mathrm{BB}}
\end{array}\right]=\left[\begin{array}{ll}
\boldsymbol{G}_{(\mathrm{HRH})} & \boldsymbol{G}_{(\mathrm{HRR})} \\
\boldsymbol{G}_{(\mathrm{RHH})} & \boldsymbol{G}_{(\mathrm{RHR})}
\end{array}\right]
$$

where, $\boldsymbol{G}_{(\mathrm{HRH})}, \boldsymbol{G}_{(\mathrm{HRR})}, \boldsymbol{G}_{(\mathrm{RHH})}$, and $\boldsymbol{G}_{(\mathrm{RHR})}$ indicate the matrices $G$ 's obtained eq 10 with $\langle\boldsymbol{T}\rangle$ expressed by eq $23,21,22$, and 20 , respectively.

In the first place, let $\mathrm{A}(i . e .$, Ala) be the minor component (i.e., $0<p_{\mathrm{A}}<0.5$ ) of the copolymer. The sequence probability $p_{A \mathrm{~A}}$ can take values of $0<p_{\mathrm{AA}}<1$. Thus $p_{\mathrm{A}}$ and $p_{\mathrm{AA}}$ are given. Then the numerical values of $p_{\mathrm{B}}, p_{\mathrm{AB}}, p_{\mathrm{BA}}$, and $p_{\mathrm{BB}}$ are explicitly determined from eq 4 and 6 . In Figure 8, the characteristic ratios for $\mathrm{Ala}(\mathrm{A})$ $-\mathrm{NMG}(\mathrm{B})$ copolymers, in which $\mathrm{A}$ is the minor component and $n=153$, are shown as a function of the sequence probability $p_{\mathrm{AA}}$. Next, letting $\mathrm{B}($ i.e., NMG) be the minor component, we obtain the results as shown in Figure 9. The numerals appearing in these figures denote mole $\%$ of Ala (Figure 8) and of NMG (Figure 9).

The characteristic ratios of equimolar AlaNMG copolymers with different chain lengths are shown in Figure 10 as a function of the sequence probability $p$, by assuming $p=p_{\mathrm{AA}}=$ $p_{\mathrm{BB}}$. The numerals in the figure indicate the

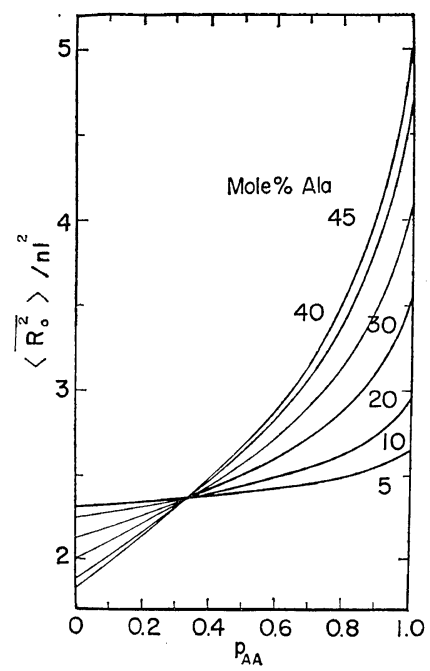

Figure 8. Plot of the characteristic ratio against the sequence probability $p_{\mathrm{AA}}$ for Ala(A)-NMG(B) copolymers $(n=153)$. The numerals on the curves denote the mol\% of $\mathrm{A}$ (minor component).

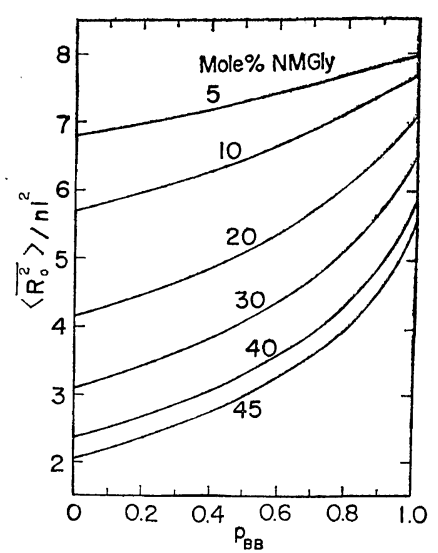

Figure 9. Plot of the characteristic ratio against the sequence probability $p_{\mathrm{B}}$ for $\mathrm{Ala}(\mathrm{A})-\mathrm{NMG}(\mathrm{B})$ copolymers $(n=153)$. The numerals on the curves denote the mol $\%$ of $\mathrm{B}$ (minor component).

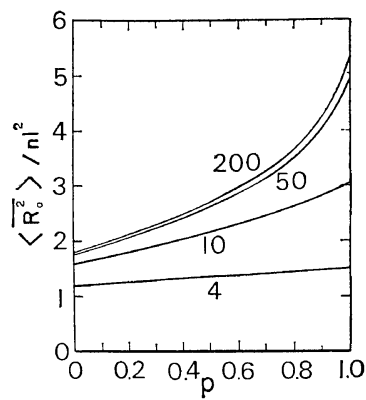

Figure 10. Plot of the characteristic ratio against the sequence probability $p\left(=p_{\mathrm{AA}}=p_{\mathrm{BB}}\right)$ for equimolar $\mathrm{Ala}(\mathrm{A})-\mathrm{NMG}(\mathrm{B})$ copolymers. The numerals on the curves denote the number of virtual bonds, $n$, in the chain.

number $n$ of the virtual bonds in the chain. The characteristic ratios at $p=0, p=0.5$, and $p=1$ correspond, respectively, to those for the alternative copolymer, the equimolar random copolymer, and the equimolar mixture of Alaand NMG-homopolymer.

(b) Copolymers of Alanine and N-methyl-Lalanine. This type of copolymer also belongs to type II. Representing Ala and NMA by A and $\mathrm{B}$, respectively, we have

$$
\left[\begin{array}{ll}
\boldsymbol{G}_{\mathrm{AA}} & \boldsymbol{G}_{\mathrm{AB}} \\
\boldsymbol{G}_{\mathrm{BA}} & \boldsymbol{G}_{\mathrm{BB}}
\end{array}\right]=\left[\begin{array}{ll}
\boldsymbol{G}_{(\mathrm{HRH})} & \boldsymbol{G}_{(\mathrm{HRR})} \\
\boldsymbol{G}_{(\mathrm{RRH})} & \boldsymbol{G}_{(\mathrm{RRR})}
\end{array}\right]
$$

The characteristic ratios of $\mathrm{Ala}(\mathrm{A})-\mathrm{NMA}(\mathrm{B})$ 


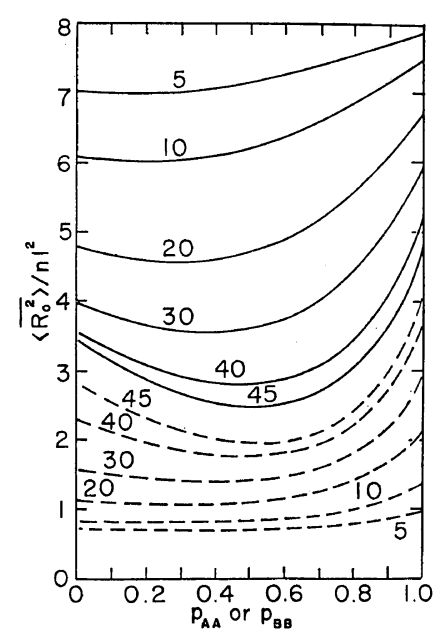

Figure 11. Plot of the characteristic ratio against the sequence probability $p_{\mathrm{AA}}$ or $p_{\mathrm{BB}}$ for $\mathrm{Ala}(\mathrm{A})-$ NMA(B) copolymers $(n=153)$.

Solid curves: $\mathrm{NMA}(\mathrm{B})$ is the minor component; numerals on the curves are mol\% of $\operatorname{NMA}(B)$; abscissa is $p_{\mathrm{BB}}$. Broken curves: $\mathrm{Ala}(\mathrm{A})$ is the minor component; numerals on the curves are mol\% of $\mathrm{Ala}(\mathrm{A})$; abscissa is $p_{\mathrm{AA}}$.

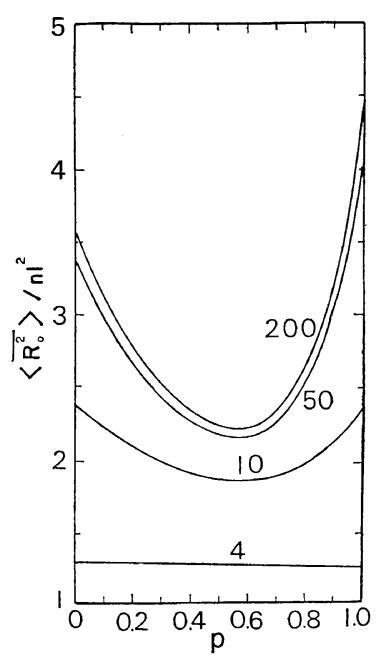

Figure 12. Plot of the characteristic ratio against the sequence probability $p\left(=p_{\mathrm{AA}}=p_{\mathrm{BB}}\right)$ for equimolar $\mathrm{Ala}(\mathrm{A})-\mathrm{NMA}(\mathrm{B})$ copolymers. The numerals on the curves denote the number of virtual bonds, $n$, in the chain.

copolymers $(n=153)$ as a function of the sequence probability are shown in Figures 11 and 12. In Figure 11, the minor components are $\mathbf{A}$ and $\mathbf{B}$, respectively; Figure 12 refers to equimolar copolymers with different chain lengths.

(c) Copolymers of Alanine and Glycine. Copolymers composed of Ala(A-component)-Gly (B-component) belong to type I. The characteristic ratio is obtained from eq 13 , by using $\boldsymbol{K}[\mathrm{eq} 11]$ constructed with $\left[\begin{array}{ll}\boldsymbol{G}_{\mathrm{A}} & \boldsymbol{G}_{\mathrm{B}}\end{array}\right]=$ $\left[\boldsymbol{G}_{(\mathrm{HRH})}, \boldsymbol{G}_{(\mathrm{HHH})}\right]$. In Figure 13, the characteristic ratios of $\mathrm{Ala}(\mathrm{A})-\mathrm{Gly}(\mathrm{B})$ copolymers $(n=$ 153) are plotted against $p_{\mathrm{AA}}(\mathrm{A}$ is the minor component) or $p_{\mathrm{BB}}$ ( $\mathrm{B}$ is the minor component). For equimolar copolymer with $n=151$, the characteristic ratio is also shown in Figure 13 as a function of $p$ on the assumption of $p=p_{\mathrm{AA}}=p_{\mathrm{BB}}$.

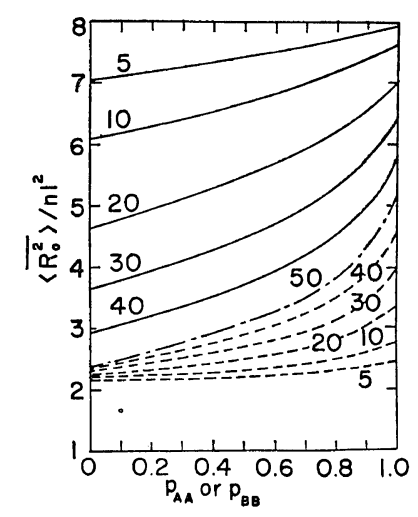

Figure 13. Plot of the characteristic ratio against the sequence probability for $\mathrm{Ala}(\mathrm{A})-\mathrm{Gly}(\mathrm{B})$ copolymers.

Solid curves: $\mathrm{Gly}(\mathbf{B})$ is the minor component and $n=153$; numerals on the curves are mol $\%$ of $\mathbf{B}$ (Gly); abscissa is $p_{\mathrm{BB}}$. Chain curve: equimolar copolymer $(n=151)$; abscissa is $p_{\mathrm{AA}}=p_{\mathrm{BB}}$. Broken curves: $\mathrm{Ala}(\mathrm{A})$ is the minor component and $n=$ 153 ; numerals on the curves are mol\% of $\mathrm{A}(\mathrm{Ala})$; abscissa is $p_{\mathrm{AA}}$.

(d) Copolymers Composed of Glycine and Lalanine, of Glycine and $N$-methyl-L-alanine, and of $N$-methylglycine and $N$-methyl-L-alanine. Gly (A)-NMG(B) and $\mathrm{Gly}(\mathrm{A})-\mathrm{NMA}(\mathrm{B})$ copolymers belong to type II, while $\mathrm{NMG}(\mathrm{A})$-NMA (B) copolymer belongs to type I. For Gly(A) - NMG(B) copolymer

$$
\left[\begin{array}{ll}
\boldsymbol{G}_{\mathrm{AA}} & \boldsymbol{G}_{\mathrm{AB}} \\
\boldsymbol{G}_{\mathrm{BA}} & \boldsymbol{G}_{\mathrm{BB}}
\end{array}\right]=\left[\begin{array}{ll}
\boldsymbol{G}_{(\mathrm{HHH})} & \boldsymbol{G}_{(\mathrm{HHR})} \\
\boldsymbol{G}_{(\mathrm{RHH})} & \boldsymbol{G}_{(\mathrm{RHR})}
\end{array}\right]
$$

for $\operatorname{Gly}(\mathrm{A})-\mathrm{NMA}(\mathrm{B})$ copolymer 


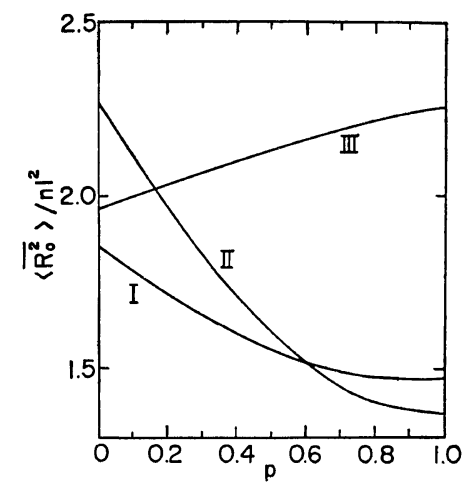

Figure 14. Plot of the characteristic ratio against the sequence probability $p\left(=p_{\mathrm{AA}}=p_{\mathrm{BB}}\right)$ for equimolar NMA-NMG copolymers (curve I), equimolar Gly-NMA copolymers (curve II), and equimolar Gly-NMG copolymers (curve III).

$$
\left[\begin{array}{ll}
\boldsymbol{G}_{\mathrm{AA}} & \boldsymbol{G}_{\mathrm{AB}} \\
\boldsymbol{G}_{\mathrm{BA}} & \boldsymbol{G}_{\mathrm{BB}}
\end{array}\right]=\left[\begin{array}{ll}
\boldsymbol{G}_{(\mathrm{HHH})} & \boldsymbol{G}_{(\mathrm{HHR})} \\
\boldsymbol{G}_{(\mathrm{RRH})} & \boldsymbol{G}_{(\mathrm{RRR})}
\end{array}\right]
$$

and for $\mathrm{NMG}(\mathrm{A})-\mathrm{NMA}(\mathrm{B})$ copolymer

$$
\left[\begin{array}{ll}
\boldsymbol{G}_{\mathrm{A}} & \boldsymbol{G}_{\mathrm{B}}
\end{array}\right]=\left[\begin{array}{ll}
\boldsymbol{G}_{(\mathrm{RHR})} & \boldsymbol{G}_{(\mathrm{RRR})}
\end{array}\right]
$$

The characteristic ratios for corresponding copolymers are obtained from eq 17 with eq 28 or 29 and from eq 13 with eq 30.

In Figure 14 are shown the characteristic ratios of equimolar copolymers $(n=150)$ composed of Gly and NMG, of Gly and NMA, and of NMG and NMA, as functions of sequence probability $p$, by assuming $p=p_{\mathrm{AA}}=p_{\mathrm{BB}}$.

(e) Racemic Copolymers of $N$-methyl-D-alanine and $N$-methyl-L-alanine. The averaged transformation matrix $\langle\boldsymbol{T}\rangle_{\mathrm{L}-(\mathrm{RRR})}$ for $N$-methyl-L-alanine is already given by eq 18 . The conformational energy $V_{\mathrm{L}(\mathrm{RRR})}(\phi, \phi)$ of $\mathrm{L}$-isomer is related to that of D-isomer by

$$
V_{\mathrm{L}(\mathrm{RRR})}(\phi, \phi)=V_{\mathrm{D}(\mathrm{RRR})}(-\phi,-\phi)
$$

Thus, the matrix $\langle\boldsymbol{T}\rangle_{\mathrm{D}(\mathrm{RRR})}$ for $N$-methyl-Dalanine is derived, from the relation just mentioned above, as

$$
\langle\boldsymbol{T}\rangle_{\mathrm{D}-(\mathrm{RRR})}=\left[\begin{array}{rrr}
0.332 & 0.132 & -0.835 \\
-0.166 & 0.552 & 0.221 \\
0.557 & 0.027 & 0.223
\end{array}\right]
$$

The racemic NMA copolymers belong to type $\mathrm{I}$; hence the characteristic ratio is obtained from eq 13 by letting

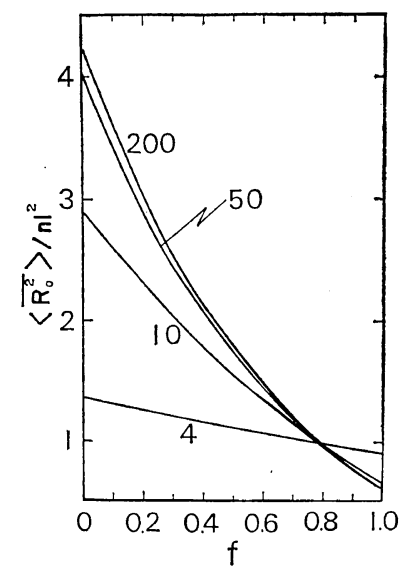

Figure 15. Plot of the characteristic ratio against the isotacticity, $f$, for racemic D,L-NMA copolymers. The numerals on the curves denote the number of virtual bonds, $n$, in the chain.

$$
\left[\begin{array}{ll}
\boldsymbol{G}_{\mathrm{A}} & \boldsymbol{G}_{\mathrm{B}}
\end{array}\right]=\left[\begin{array}{ll}
\boldsymbol{G}_{\mathrm{L}-(\mathrm{RRR})} & \boldsymbol{G}_{\mathrm{I})-(\mathrm{RRR})}
\end{array}\right]
$$

in eq 11. In the polymerization of $\mathrm{D}$ and $\mathrm{L}$ units, for the present system, the probability $p_{A \mathrm{~A}}$ that a D-residue follows a D-residue is assumed to be equal to that $p_{\mathrm{LL}}$ for an L-residue to follow an L-residue, and represented as $p_{\mathrm{LL}}=p_{\mathrm{DD}}=$ $f$. $f$ is regarded as a measure of isotacticity of the chain. The results of calculations are shown in Figure 15, in which the numerals denote the number $n$ of the virtual bonds in the chain. The characteristic ratios at $f=0, f=0.5$, and $f=1$ correspond to those for completely syndiotactic polymer, random D,L-copolymer, and an equimolar mixture of D- and L-homopolymers.

(f) D,L-copolymers of Alanine or of $N$-methylalanine. In this section, we are concerned with copolymers containing D- and L-enantiomorphs in different molar ratios. The case where the symmetry principle, i.e., $p_{\mathrm{LL}}=p_{\mathrm{DD}}$ and $p_{\mathrm{LD}}=p_{\mathrm{DL}}$, holds in the polymerization process, has already been discussed in section (e). In the copolymerization of $\mathrm{D}$ - and $\mathrm{L}-\mathrm{N}$-carboxyanhydrides, the relative reactivities of the components may depend not only on the terminal residue of the growing chain but also on a particular residue preceded by a few (two or three) residues from the terminal residue. In such a case, the chain may be treated ${ }^{7}$ as a Markoffian chain which satisfies given conditions. In principle, this may be performed by increasing the order of the 
matrix $\boldsymbol{P}$. However, in the present treatment, D,L-copolymers are regarded as obeying the simple Markoffian process in which reactivities of the components depend only on the terminal residue of the growing chain. The copolymers referred to here are regarded as those polymerized under conditions in which the symmetry principle is broken, i.e., $p_{\mathrm{LL}} \neq p_{\mathrm{DD}}$.

D,L-Ala-copolymers and D,L-NMA-copolymers belong to type $\mathrm{I}$. The averaged transformation matrix $\langle\boldsymbol{T}\rangle_{\mathrm{D}-(\mathrm{HRH})}$ for D-alanine is derived from

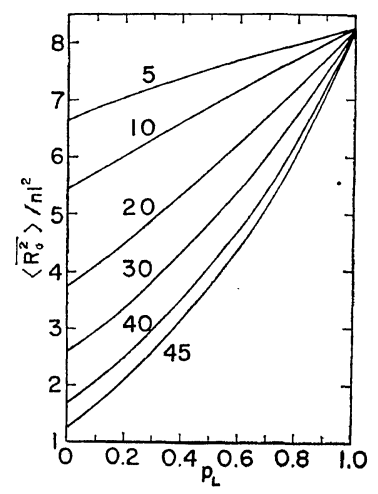

Figure 16. Plot of the characteristic ratio against the sequence probability $p$ that a residue of the minor component is succeeded by the same kind residue for $\mathrm{D}, \mathrm{L}-\mathrm{Ala}$ copolymers $(n=150)$. The numerals on the curves denote the mol\% of the minor component.

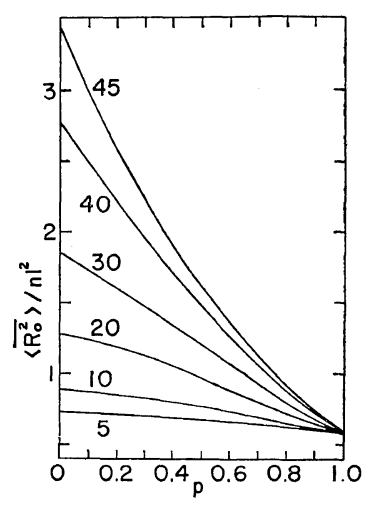

Figure 17. Plot of the characteristic ratio against the sequence probability $p$ that a residue of the minor component is succeeded by the same kind residue for $\mathrm{D}, \mathrm{L}-\mathrm{NMA}$ copolymers $(n=150)$. The numerals on the curves denote the mol $\%$ of the minor component. eq 23 for L-alanine:

$$
\langle\boldsymbol{T}\rangle_{\mathrm{D}-(\mathrm{HRH})}=\left[\begin{array}{rrr}
0.565 & 0.370 & -0.499 \\
-0.055 & -0.573 & -0.417 \\
-0.566 & 0.417 & -0.330
\end{array}\right]
$$

The characteristic ratios are calculated from eq 13 by using $K$ constructed from

$$
\left[\begin{array}{ll}
\boldsymbol{G}_{\mathrm{A}} & \boldsymbol{G}_{\mathrm{B}}
\end{array}\right]=\left[\begin{array}{ll}
\boldsymbol{G}_{\mathrm{L}-(\mathrm{HRH})} & \boldsymbol{G}_{\mathrm{D}-(\mathrm{HRH})}
\end{array}\right] \text { for Ala, }
$$
and

$$
\left[\begin{array}{ll}
G_{\mathrm{A}} & \boldsymbol{G}_{\mathrm{B}}
\end{array}\right]=\left[\begin{array}{lll}
\boldsymbol{G}_{\mathrm{L}-(\mathrm{RRR})} & \boldsymbol{G}_{\mathrm{D}-(\mathrm{RRR})}
\end{array}\right] \text { for NMA. }
$$

The characteristic ratio, as a function of the sequence probability $p\left(\right.$ i.e., $p_{\mathrm{LL}}$ or $\left.p_{\mathrm{DD}}\right)$ that a residue of the minor component is succeeded by the same kind of residue, is shown in Figure 16 for D,L-Ala-copolymers, and in Figure 17 for D,L-NMA-copolymers. Note that $p_{\mathrm{LL}} \neq p_{\mathrm{DD}}$ The numerals on the curves indicate the mole $\%$ of the minor component; for example, the abscissa gives $p_{\mathrm{LL}}$ for $\mathrm{D}: \mathrm{L}=80: 20$ copolymer, while it gives $p_{\mathrm{DD}}$ for $\mathrm{D}: \mathrm{L}=20: 80$ copolymer. These figures lead to the plots of characteristic ratio against the stereocomposition (say $\% \mathrm{~L}-$ isomer) of randomly sequenced $\mathrm{D}, \mathrm{L}-\mathrm{Ala}$ - or $\mathrm{D}, \mathrm{L}-$ NMA-copolymer which give a symmetrical curve about an axis at $\% \mathrm{~L}=50$.

Effect of Helical Parts on $\left\langle R_{0}{ }^{2}\right\rangle / n l^{2}$ of Binary Copolymers Containing N-Methylalanine

In a preceding paper, ${ }^{1}$ we pointed out that the $\operatorname{poly}(N$-methylalanine $)$ chain is extremely restricted for rotations about the $\mathrm{N}-\mathrm{C}^{\alpha}$ and $\mathrm{C}^{\alpha}-$ $\mathrm{C}^{\prime}$ bonds. In a contour diagram, four conformationally available regions, I, II, III, and IV, were found. In particular, the contribution of the region III containing the left-handed $\alpha$-helix, is very large. ${ }^{1}$ The calculated characteristic ratio for infinite chain is unusually as small as 0.6. Neither the circumstances where the NMA chain portion exists either in helix or in coil conformation, nor information about the helix -coil transition for NMA chains has been ascertained experimentally as yet. However, there is a possibility that the NMA chain portion in homopolymers and copolymers takes a helix conformation. If the NMA chain takes a crystalline structure even in solution, then the probable conformation is assumed to be lefthanded $\alpha$-helix. 


\section{S. TANAKA and A. NAKaJima}

In this section we shall discuss the effect of the rod-like portion of the helix conformation on the characteristic ratio. Such considerations are similar to those reported by $\mathrm{Nagai}^{11}$ on the change of the characteristic ratio in the helixcoil transition region. Nagai's treatment is based on the equilibrium theory. On the other hand, in the present treatment, the NMA residue is treated as contributing only to the helix.

Our calculations done on the copolymer composed of Ala and NMA, as an example, assumed that Ala-residues were in random coil and NMA-residues were in left-handed $\alpha$-helix conformation. The rotational angles $\phi, \phi$ for NMA were assumed to be $\phi=-122^{\circ}, \phi=-133^{\circ}$, as assigned to the left-handed $\alpha$-helix by Pauling and Cory, ${ }^{12}$ although the lowest energy point obtained from the contour diagram was located at $\phi=-120^{\circ}, \phi=-100^{\circ}$ (see Figure 6 in ref 1). Let the transformation matrix for NMA in the left-handed $\alpha$-helix conformation be $\boldsymbol{T}_{\text {helix }}$. In section $(b)$, Ala-NMA copolymers were treated by assuming that both Ala and NMA are in random coil conformation. For the present model, $\boldsymbol{G}_{\text {heiix }}$ was constructed from eq 10 by using $\boldsymbol{T}_{\text {helix }}$ in place of $\langle\boldsymbol{T}\rangle$. The obtained $\boldsymbol{G}_{\mathrm{helix}}$ was inserted in eq 27 in place of $\boldsymbol{G}_{(\mathrm{RRR})}$, to obtain the following equation.

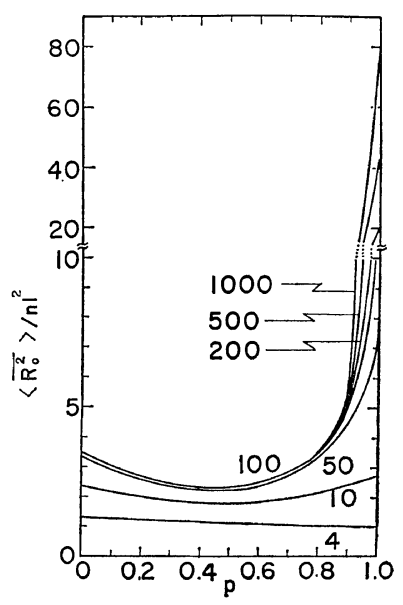

Figure 18. Plot of the characteristic ratio, calculated by assuming NMA residues in helix conformation, against the sequence probability $p$ for equimolar Ala-NMA copolymers. The numerals on the curves denote the number of virtual bonds, $n$, in the chain.

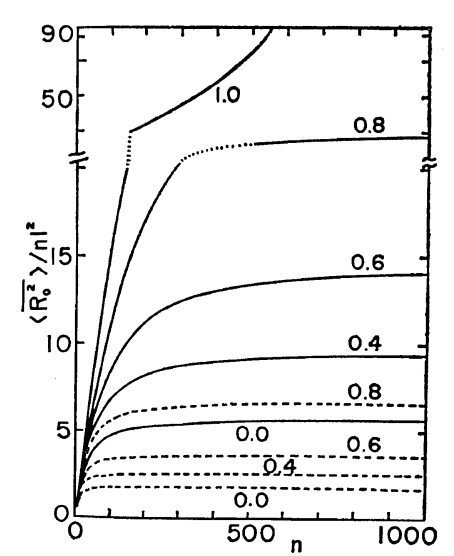

Figure 19. Plot of the characteristic ratio, calculated by assuming NMA residues in helix conformation, against the number of virtual bonds, $n$, for Ala-NMA copolymers.

Solid curves: Ala content is $5 \mathrm{~mol} \%$. Broken curves: Ala content is $20 \mathrm{~mol} \%$. The numerals on the curves denote the sequence probability of Ala-Ala.

$$
\left[\begin{array}{ll}
\boldsymbol{G}_{\mathrm{AA}} & \boldsymbol{G}_{\mathrm{AB}} \\
\boldsymbol{G}_{\mathrm{BA}} & \boldsymbol{G}_{\mathrm{BB}}
\end{array}\right]=\left[\begin{array}{ll}
\boldsymbol{G}_{(\mathrm{HRH})} & \boldsymbol{G}_{(\mathrm{HRR})} \\
\boldsymbol{G}_{(\mathrm{RRH})} & \boldsymbol{G}_{\mathrm{helix}}
\end{array}\right]
$$

The characteristic ratio for a copolymer with finite chain length was calculated from eq 17 using $\boldsymbol{K}$ derived from eq 33.

In Figure 18, the characteristic ratios for equimolar Ala-NMA copolymers with various $n$ are shown as a function of $p$. The dependence of the characteristic ratios on $n$, the number of virtual bonds in the chain, is shown in Figure 19 , where solid curves are concerned with 5mol\% Ala and broken curves with 20-mol\% Ala content. The numerals on the curves denote the values of sequence probability $p_{\mathrm{AA}}$ that an Ala unit is followed by an Ala unit.

The effect of the junction where the helix and coil meet as treated in eq 33 , is not taken into consideration. This effect may appear in $\boldsymbol{G}_{(\mathrm{HRR})}$ and $\boldsymbol{G}_{(\mathrm{RRH})}$. But it does not seem large, owing to the absence of the effect of hydrogen bonding.

\section{DISCUSSION}

In this paper, the characteristic ratios are investigated on various types of copolypeptides. 
In a following paper we shall discuss some characteristic features of the results obtained.

We have referred to the copolymers containing alanine residues, e.g., Ala-NMG copolymers (Figures 8-10), Ala-NMA copolymers (Figures 11 and 12), and Ala-Gly copolymers (Figure 13). To compare these three copolymers, it is convenient to use Figures 10, 12, and 13, obtained for corresponding equimolar copolymers. With respect to the relation between the characteristic ratio and the sequence probability $p$, the characteristic ratios for both the Ala-NMG (Figure 10) and Ala-Gly (Figure 13) copolymers increase with increasing $p$, while those for Ala-NMA copolymers (Figure 12) have a minimum at $p=0.5$, i.e., for random copolymers.

The value of $\left\langle\bar{R}_{0}{ }^{2}\right\rangle / n l^{2}$ for Ala-NMA copolymers with $n=200$, in the limit of the perfectly alternating polymer $(p=0)$, is as large as 3.5 . This fact is understandable from the contour diagrams for $V_{(\mathrm{HRR})}(\phi, \phi)$ (Figure 5) and for $V_{(\mathrm{RRH})}(\phi, \phi)$ (Figure 4). Namely, for $V_{\mid \mathrm{HRR})}(\phi, \phi)$ the energy minimum locates at $\phi=20^{\circ}, \phi=340^{\circ}$, so that a rather extended conformation is preferred, on the other hand, for $V_{(\mathrm{RRH})}(\phi, \phi)$ where the minimum locates at $\phi=240^{\circ}, \phi=0^{\circ}$. In accordance with the three state model (in which trans, gauche, and another gauche conformation are, respectively, denoted by $T, G$, and $G^{\prime}$ ), it may be said that the contribution of $T T$ is large for $V_{\langle H R R\rangle}(\phi, \phi)$, and that of $G^{\prime} T$ is large for $V_{(\mathrm{RRH})}(\phi, \psi)$. These two contributions may reflect on the rather large characteristic ratio for the alternating copolymer. In the range of $0<p<0.5$, the characteristic ratios decrease with increasing $p$. This behavior may be mainly due to the fact that the influence of $V_{(R R R)}(\phi, \psi)$ (see Figure 6 in ref 1 ) is large. After the minimum is passed, the characteristic ratios in the range of $0.5<p<1$ increase with the increased blockness of the chains, which is attributed to the fact that extended conformation is preferred for the Ala-Ala sequence (see Figure 4 in ref 1). Note that the characteristic ratio of the Ala-homopolymer is as high as 8.4.

With respect to Ala-NMG copolymers, it should be noted that the characteristic ratio (1.8) of the alternating copolymer $(p=0)$ with $n=200$ (Figure 10) is less than those of 8.4 and
2.97, for the Ala-homopolymer and NMG-homopolymer, respectively. Conformation of the perfectly alternating Ala-NMG copolymer is given by merely $V_{(\mathrm{HRR})}(\phi, \psi)$ and $V_{(\mathrm{RHH})}(\phi, \psi)$ and is no longer affected by such conformations as $V_{(R R H)}(\phi, \psi)$ and $V_{(\mathrm{HRH})}(\phi, \psi)$ which contribute to NMG-homopolymers and Ala-homopolymers. For this reason, the characteristic ratio of the alternating Ala-NMG copolymer stands apart from that of the component homopolymers. From Figures 5 and, in particular, 4, it is known that the conformationally preferred regions $I$, $\mathrm{I}^{\prime}$, III, and $\mathrm{III}^{\prime}$ for $V_{\left(\mathrm{RH}_{\mathrm{H}}\right)}(\phi, \psi)$ (Figure 6) correspond to the skewed conformations as $G G^{\prime}$, $G^{\prime} G, G G$, and $G^{\prime} G^{\prime}$, which result in such a small value as 1.8 for the characteristic ratio of the alternating copolymer. This value 1.8 is very close to 1.9 for a free rotation chain. Such tendency is similar to the case of the Glyhomopolymer. ${ }^{1}$ The characteristic ratio in the range of $0.5<p<1$ montonously increased with an increasing $p$, i.e., with increasing blockness. Such behavior may be caused by the contribution of extended conformation due to Ala-Ala blockness.

In contrast to type II copolymers, type I copolymers retain the conformations characteristic to the component homopolymers. Hence, for type I copolymers, their behavior was markedly different from that presumed from component homopolymers and was not expected. Such examples are shown for Ala-Gly copolymers in Figure 13. What can be said in common about Ala-NMG, Ala-NMA, and Ala--Gly copolymers, is that the slight increase in the blockness at high $p$ abruptly increases the characteristic ratio, owing to the fact that relatively extended conformation is preferred for the Ala - Ala sequence.

The characteristic ratios of Gly-NMG copolymers, Gly-NMA copolymers, and NMG-NMA copolymers are shown in Figure 14. For NMA -NMG copolymers (curve I) and Gly-NMA copolymers (curve II), the average chain dimensions decrease as the sequence probability $p$ is increased, in contrast to Gly-NMG copolymers (curve III) for which the average chain dimensions invariably increase with $p$. The skewed conformation of the NMA residue may be re- 


\section{S. TANAKA and A. NAKajima}

sponsible for a decrease in the characteristic ratio with increasing $p$ as exhibited in curves $I$ and II. Further, the result that $\left\langle\bar{R}_{0}{ }^{2}\right\rangle / n l^{2}=2.25$ for Gly-NMA alternating copolymer $(p=0)$ is higher than those for Gly- and NMA-homopolymers $(2.17$ for Gly-, and 0.6 for NMA-homopolymer) may be attributed to the fact that this copolymer belongs to type II, so that the conformations for component homopolymers are not reflected in the copolymer. In other words, as is obvious from eq 29 , the characteristic ratios of this alternating copolymer are obtained from $V_{(\mathrm{RRH})}(\phi, \psi)$ (see Figure 4$)$ and $V_{(\mathrm{HHR})}(\phi$, $\phi)$ (see Figure 7) without being affected by $V_{(\mathrm{HHH})}(\phi, \psi)$ and $V_{(\mathrm{RRR})}(\phi, \psi)$. In particular, it is noted that the energy minimum position in Figure 7 locates at $\phi=100^{\circ}$ and $\phi=0^{\circ}$.

A feature in $\left\langle\bar{R}_{0}{ }^{2}\right\rangle / n l^{2}$ of the racemic NMAcopolymers is that the characteristic ratio is the largest for alternating $\mathrm{D}, \mathrm{L}$-copolymer $(f=0)$, and monotonously decreases with an increasing $f$ in the range of $0<f<1$ (Figure 15), in contrast to racemic Ala-copolymers where the characteristic ratio, as pointed out by Miller, et al., ${ }^{2}$ monotonously increases with $f$. As pointed out in a preceding paper, ${ }^{1}$ the L-NMA-homopolymer takes compact conformation. This is true also for the D-NMA-homopolymer. However, in the alternating D,L-NMA-copolymer, conformations correspond to the mirror image of each other appearing alternatively in the chain, which leads to a relatively large value such as 4.3 for the characteristic ratio. As the isotacticity $f$ approaches 1 , the average chain becomes a compact coil peculiar to L- and D-homopolymers. Such a tendency is still retained for copolymers indicated in Figures 16 and 17.

As mentioned above, NMA residues are also treated as in helix conformation. Figures 18 and 12, obtained for equimolar Ala-NMA copolymers, show the results calculated by assuming, respectively, helix and random coil conformation for NMA residues. A comparison of these two Figures makes us understand the effect of rod-like parts on the average chain dimension. In the range $0<p<0.9$, relations between the characteristic ratio and sequence probability are not markedly different for either case in which helix or coil conformation is as- sumed for NMA residues. This is attributed to the characteristic ratio of the $\alpha$-helix portion composed of NMA residues less than 60 and is not significantly different from or smaller than that of the random coil chain. However, in the range of high blockness $(0.9<p<1)$, behaviors of both curves are different; the characteristic ratio in Figure 18 increases abruptly, because the length of the helix portion (number of residues in helix portion) increases. Moreover, as is obvious from Figure 19, the characteristic ratio of NMA-Ala copolymers is largely affected by the sequence probability, $p$ and the degrees of polymerization, $n+1$, when a small amount $(5 \%)$ of Ala is contained in the copolymer. However, with copolymers of 20$\%$ Ala content, the characteristic ratio is not largely affected by the degrees of polymerization even at $p=0.8$. For both cases $(5-\%$ and $20-\%$ Ala-copolymers), the characteristic ratios are, if the degrees of polymerization are less than 60 , not largely affected whichever helix or coil conformation is assumed for NMA residues. The reason for their behavior is due to the fact that the characteristic ratio for the shorter helix $(n<$ 60 ) is smaller than that for the corresponding random coil chain. Such a result is similar to that pointed out by $\mathrm{Nagai}^{11}$ with respect to the change of the characteristic ratio in the helixcoil transition region.

In all the calculations mentioned above, the imide bond in NMG or NMA residue was assumed to be in trans conformation. As mentioned elsewhere, ${ }^{1,14}$ the presence of cis bonds has also been suggested. ${ }^{15}$ The effect of cis bonds on the characteristic ratio is now under consideration. Along with this, some experimental studies relating to the present paper are also being undertaken by our research group.

The computations were carried out on the KDC-II and the FACOM-60 computers at the Kyoto University Computation Center. This work was presented at the SPSJ Microsymposium 1970-I in Kyoto, on November 21, 1970.

\section{REFERENCES}

1. S. Tanaka and A. Nakajima, Polymer J., 2, 717 (1971).

2. W. I. Miller, D. A. Brant, and J. P. Flory, $J$. 


\section{Chain Conformation of Polypeptide Copolymers}

Mol. Biol., 23, 67 (1967); P. R. Schimmel and P. J. Flory, ibid., 34, 105 (1968).

3. P. J. Flory, "Statistical Mechanics of Chain Molecules"' Interscience Publishers, New York, N.Y., 1969, Chap. VII.

4. D. A. Brant and P. J. Flory, J. Amer. Chem. Soc., 87, 2791 (1965).

5. G. Némethy and H. A. Scheraga, Biopolymers, 3, 155 (1965).

6. D. A. Brant, W. G. Miller, and P. J. Flory, J. Mol. Biol., 23, 47 (1967).

7. L. Peller, J. Chem. Phys., 43, 2355 (1965).

8. D. Poland and H. A. Scheraga, "Theory of Helix-Coil Transition in Biopolymers," Aca- demic Press, New York, N.Y., 1970, p 138.

9. P. J. Flory, Proc. Natl. Acad. Sci., 51, 1060 (1964).

10. P. J. Flory and R. L. Jernigan, J. Chem. Phys., 42, 3509 (1965).

11. K. Nagai, J. Chem. Phys., 34, 887 (1961).

12. L. Pauling and R. B. Corey, Proc. Natl. Acad. Sci. USA., 37, 729 (1951).

13. S. Tanaka and A. Nakajima, Polymer J., 1, 505 (1970).

14. S. Tanaka and A. Nakajima, Polymer J., 1, 71 (1970).

15. F. A. Bovey, J. J. Ryan, and F. P. Hood, Macromolecules, 1, 305 (1968). 